\title{
NANOLITER-VOLUME DISCRETE DROP INJECTION AND PUMPING IN MICROFABRICATED CHEMICAL ANALYSIS SYSTEMS
}

\author{
Kalyan Handique ${ }^{1}$, David T. Burke ${ }^{2}$, Carlos H.Mastrangelo ${ }^{3}$ and Mark A.Burns ${ }^{1 *}$ \\ ${ }^{1}$ Department of Chemical Engineering \\ ${ }^{2}$ Department of Human Genetics \\ ${ }^{3}$ Department of Electrical Engineering and Computer Science \\ The University of Michigan, \\ 2300 Hayward Street, Rm. 3029, Ann Arbor, MI 48109 \\ Phone: (313) 763 3078, Fax: (313) 7630459 \\ *Email: maburns@umich.edu
}

\begin{abstract}
This paper presents the combined use of selective hydrophobic patterns and on-chip pressure generation for injection, pumping, mixing, and positioning of discrete sample drops in a microfabricated chemical analysis device. The device consisting of a glass substrate bonded to a silicon/quartz substrate has been fabricated and tested. Specfic regions of the etched microchannel network are selectively patterned to be hydrophobic. These hydrophobic regions are used to control the location of liquid inside the microchannel network. Precise nanoliter-volume liquid samples are split-off and moved by heating air trapped in microfabricated chambers. Discrete sample drops of the order of $35 \mathrm{nl}$ have been injected, moved, mixed and positioned in the device. This device demonstrates a simple valveless technique for performing microfluidic operations without the use of mechanical moving parts.
\end{abstract}

Keywords: Discrete Drop, Injection, Pumping, Hydrophobic Patterns, On-chip Pressure.

\section{INTRODUCTION}

Injection, motion, and positioning of discrete drops is crucial in microfabricated chemical analysis systems $[\mathbf{1 , 2}]$. The mixing, reaction, and separation steps performed in these systems require precise volumes of liquid samples to be accurately moved and positioned in the micro-channel network $[1,2]$. Although mechanical valves and pumps have been reported in literature $[\mathbf{3 , 4}]$, combinations of these have been mostly used for pumping continuous liquid samples. Discrete pumping of liquid samples has been shown using thermocapillary pumping [2] and using air pressure regulated by external pneumatic valves $[\mathbf{1 , 5}]$. In our earlier work, we have shown splitting of a nanoliter-volume discrete drop using external air pressures and selective hydrophobic patches in microchannels [5]. We also demonstrated the on-chip generation of air pressure required for drop splitting [5]. In this paper, we will show the use of selective hydrophobic patterns and on-chip pressure generation to perform the microfluidic operations of discrete drop injection, motion, mixing and positioning.

Typical microfluidic operations in microfabricated chemical analysis systems involve injection of liquid samples and reagents into microchannels. The liquid samples are mixed and moved to the "reaction" stage of the device. After performing a reaction, the liquid sample drop is then moved to the next stage of the device for detection and analysis of the reaction products. Figure 1 shows the schematic of such a device which consists of a microchannel network with specific regions selectively patterned hydrophobic. The device comprises two main channels merging into one with appropriate overflow channels and vents intersecting the main channel. During operation, liquid placed at the inlet gets drawn into the channel by capillary action and stops at the hydrophobic patch. After positioning the liquid samples, definite-sized discrete drops are split-off by use of external pressure supplied through hydrophobic air lines. The volume of each drop is defined by the channel cross-sectional area and the distance from the splitting air line to the hydrophobic interface. The two drops merge at the $y$-intersection and are positioned beyond the hydrophobic vent. The drop may be moved again by use of air pressures after closing the vent.

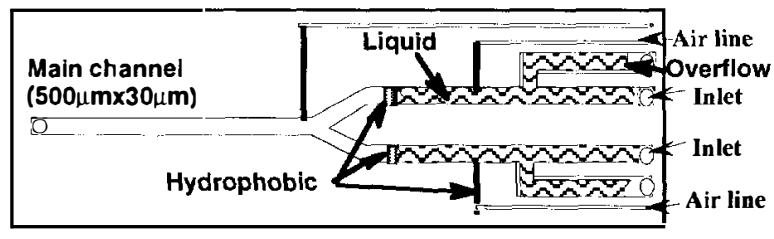

(a)

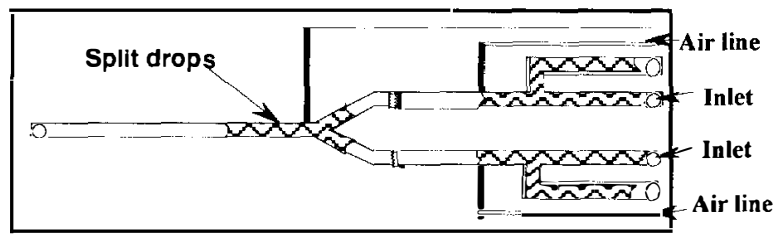

(b)

Figure 1. Schematic of a device to perform injection, mixing and positioning of discrete liquid samples. The microchannel network contains hydrophobic patches for stopping the liquid. Hydrophobic air lines are also used for pumping discrete drops and the vents are used for positioning the drops. (a): Water placed at the inlet gets drawn into the microchannel and is stopped at the hydrophobic interface. (b): Pressurizing the air lines splits off two discrete sample drops, merges them and positions them beyond the hydrophobic vent.

The pressure required for splitting and moving of discrete drops can also be generated by heating air trapped in 
strategically located microfabricated chambers. We have demonstrated the use of on-chip pressure generation for the injection of a single drop [5]. Figure 2 shows the schematic of a device similar to Figure 1 with an added ability to generate the required air pressure on-chip. Air chambers are connected to the main channel through the splitting channel and heaters placed beneath the chamber heat the air inside the chamber to increase the air pressure. This heating, in effect, splits off two discrete sample drops and moves them. The two drops are then mixed at the $y$-intersection and positioned beyond the hydrophobic vent. Cooling the air chamber slowly allows air to flow from outside and re-establish atmospheric pressure inside the chamber. The discrete drop may then be moved again by reheating the air chambers after having closed the vent line.

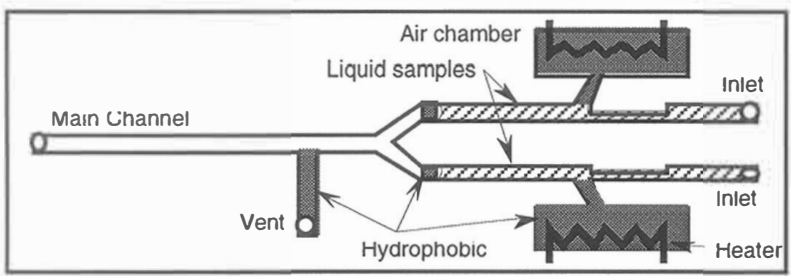

(a)

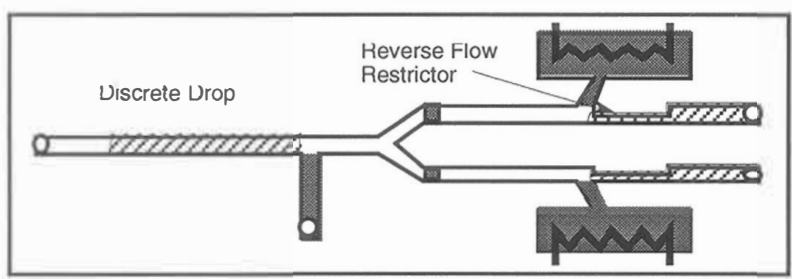

(b)

Figure 2. Schematic of a device to perform microfluidic operations using air chambers for on-chip pressure generation.

(a): Liquid samples placed at the inlet stop at the hydrophobic patches. (b): Actuation of the heaters increases the temperature, and hence pressure, inside the chamber. The subsequent air expansion splits off two discrete sample drops, mixes them at the $y$-intersection and positions the combined drop beyond the hydrophobic vent.

\section{MODELING}

The ability of an air chamber to maintain a certain gauge pressure will depend on the chamber volume. The air chamber volume and the heat required to achieve specific pressures can be estimated by modeling the dynamics of the air flowing out of a heated air chamber (Figure 3a). The chamber is maintained at room temperature initially. At time $t=0$, heat is supplied to raise the temperature (and pressure) of air in the chamber and induce convective air flow out of the chamber.

The heat and mass balance equations are solved after making some simplifying assumptions that include [6]: the gas is assumed to be ideal; the power supplied heats only the air inside the chamber; there are no entrance and exit effects in the flow of gas; the pressure and temperature is uniform inside the chamber; and, the flow rate of air maintained in the channel is proportional to the pressure drop. Chambers should be able to generate a few inches of water and maintain this pressure during the time of operation (a few seconds) in order to move a drop. From the modeling results (Figure 3), chamber volumes of the order of $0.1 \mathrm{~mm}^{3}$ should be sufficient.

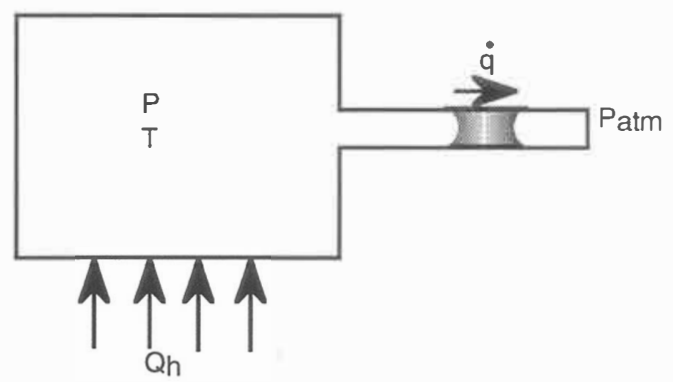

(a)

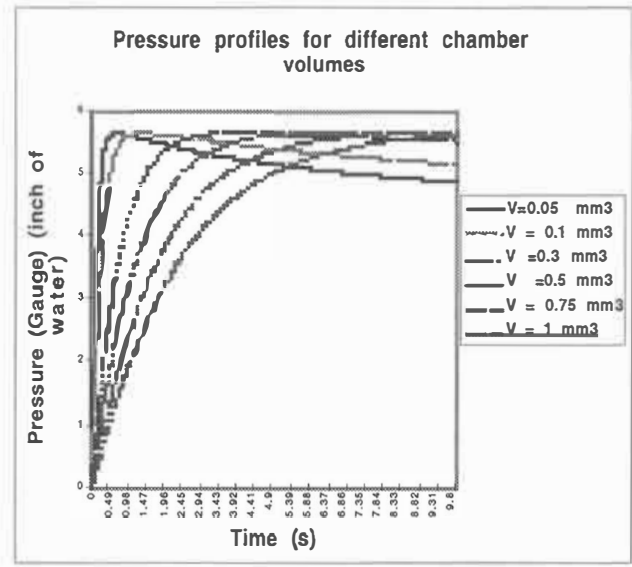

(b)

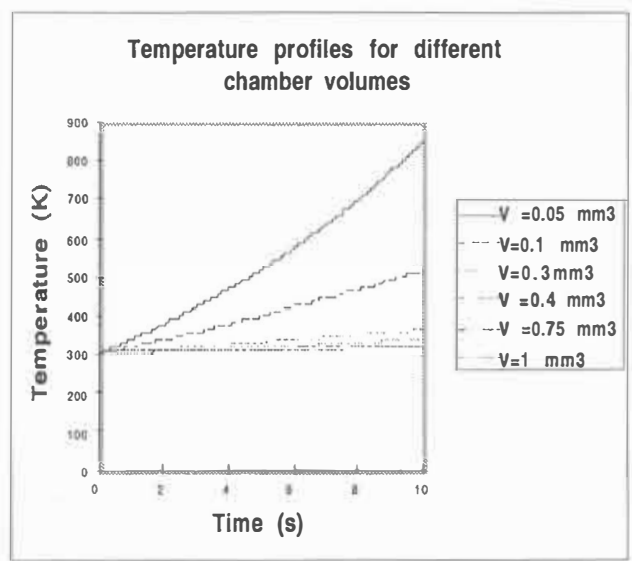

(c)

Figure 3.(a). Diagram of a chamber connected to a channel. Heat supplied to the chamber causes the air inside to expand and thus provide the pressure drop required for discrete drop motion; (b): Gauge pressure versus time for different chamber volumes for constant power $(2 \mu \mathrm{W})$ and flow proportionality constant (1000 inch water. $\left./\left(\mathrm{mm}^{3}\right)\right)$; (c):Temperature profiles obtained inside the chamber under the same conditions as 3(b). 


\section{DEVICE FABRICATION}

The device consists of a glass top containing etched chambers bonded to a silicon or quartz substrate containing metal resistive heaters and resistive temperature detectors. The fabrication steps have been described earlier [5]. For these experiments, the device has been patterned hydrophobic using a self assembled monolayer of $1 \mathrm{H}, 1 \mathrm{H}, 2 \mathrm{H}, 2 \mathrm{H}$-perfluorodecyltrichlorosilane (FDTS) [7]. Figure 4 and 5 shows the diagram and cross section of the device respectively.

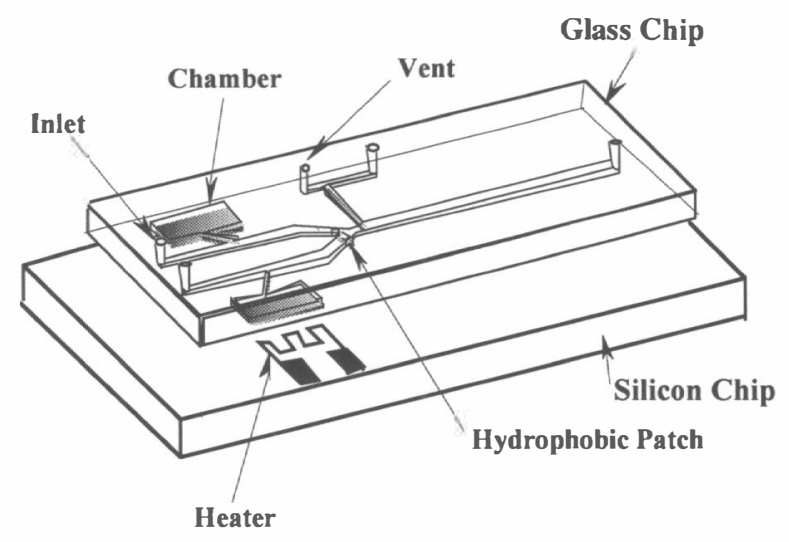

Figure 4. Diagram of the fabricated device which consists of a glass chip bonded to a Silicon/Quartz chip. Channels and chambers are etched onto the glass side. The heaters and Resistive Temperature Detectors (RTD) are fabricated on the silicon/quartz chip.

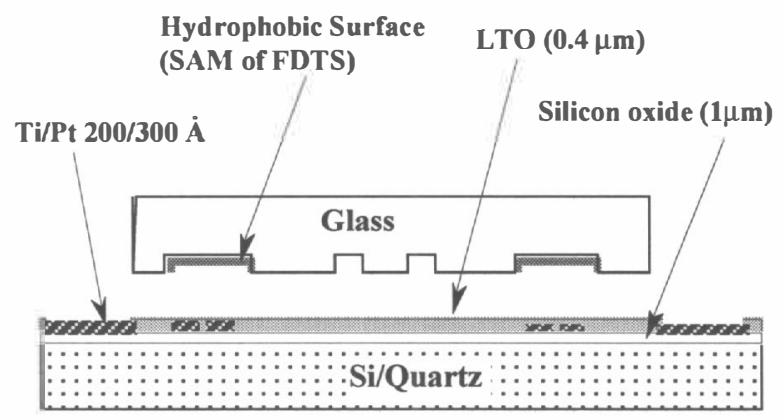

Figure 5. Cross-section of the fabricated device shown in Figure 4.

\section{RESULTS AND DISCUSSIONS}

\section{Use of External Air Pressure}

Figure 6 shows photographs of the device described earlier in Figure 1. It has a glass cap bonded to a silicon chip. The microchannels etched onto glass have a cross-section of $500 \mu \mathrm{m}$ by $30 \mu \mathrm{m}$. Water placed at the inlet by a sequencing pipet (Sigma, least count $0.5 \mu \mathrm{l}$ ) was drawn in and stopped at the hydrophobic patches. The excess water filled the overflow channel but avoided the hydrophobic air lines. The air lines were then pressurized (a few inches of water) causing two discrete-sized drops ( $100 \mathrm{nl}$ each) to split off and merge at the $y$-intersection and get positioned beyond the air vent. The discrete drop could be moved further by closing the air vent.

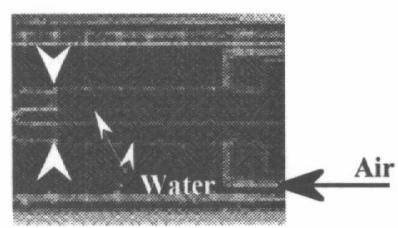

(a)

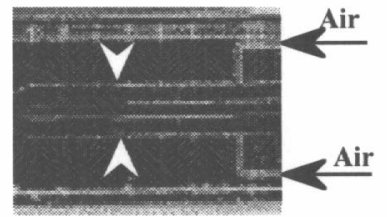

(b)
Figure 6. Demonstration of injection, motion mixing, and positinoning of discrete drops by the use of external air pressure. (a): Water placed at the inlets stops at the hydrophobic interfaces. (b): Two discrete sized drops ( $100 \mathrm{nl}$ each) split off and mix at the channel intersection.

\section{On-Chip Air Pressure Generation}

Figure 7 shows the device fabricated to perform microfluidic operations by generating the air pressures on-chip. We have performed experiments with chamber volumes ranging from $0.1 \mathrm{~mm}^{3}$ to $0.4 \mathrm{~mm}^{3}$. The main channel has a dimension of $300 \mu \mathrm{m}$ by $30 \mu \mathrm{m}$. The channels between the splitting channel and the inlet holes is narrowed down to a cross-section of 100 $\mu \mathrm{m}$ by $30 \mu \mathrm{m}$ so that the pressure generated by the air chamber is directed towards the left of the chamber.

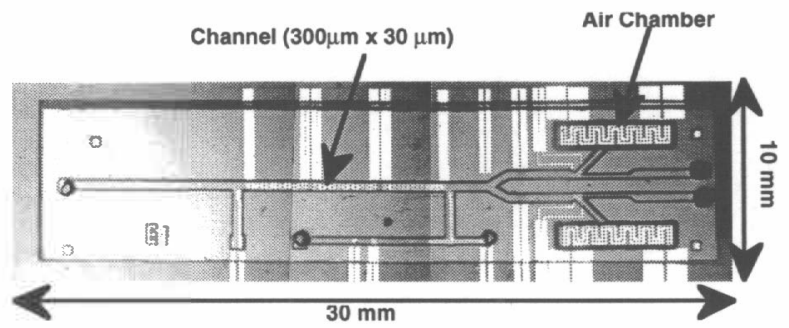

Figure 7. Photograph of the fabricated device. the chamber has a volume of $0.4 \mathrm{~mm}^{3}$.

Figure 8 shows photographs and schematics of the microfluidic operations performed. Water placed at the inlet holes stop at the hydrophobic patches. The metal heaters beneath the air chambers have a resistance of 50-56 ohms. Actuating the heaters with 4 volts causes the temperature and hence the pressure inside the chambers to rise. As a result, two drops ( $35 \mathrm{nl}$ each) are split off and moved beyond the hydrophobic patches. The drops merge and stop just beyond the hydrophobic vent. The air flowing out of the chambers flows out through the vent and ceases to push the drops further. The two drops moved a distance of $5 \mathrm{~mm}$ each in 8 seconds. The voltage applied to the heaters is then gradually reduced to zero in 4 seconds. This causes the temperature inside the chamber to 
gradually reach room temperature and thereby avoiding a sharp drop in the pressure inside the chamber. Some microfluidic operations such as a thermal reaction may be performed before the discrete drop is moved again in the micro-channel. The vent channel is then filled with water and the heaters actuated again to push the discrete drop further down the channel.

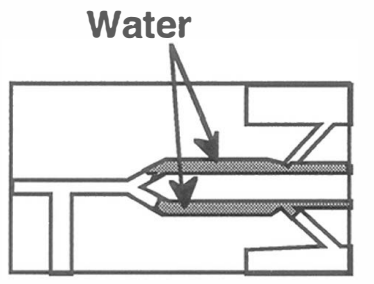

(a)
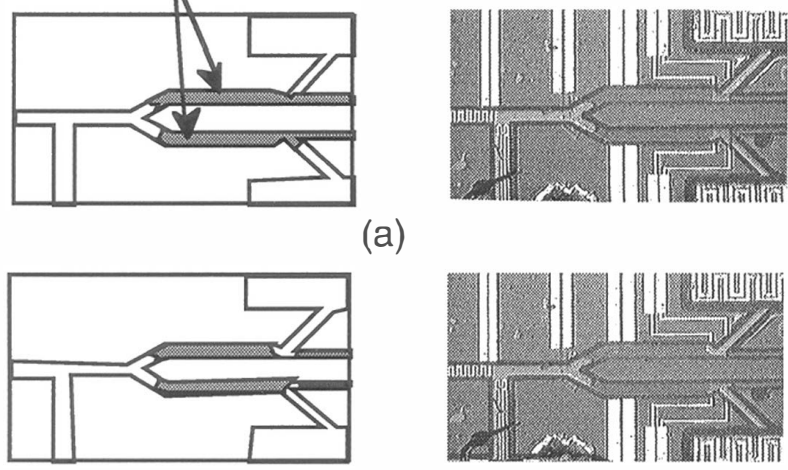

(b)
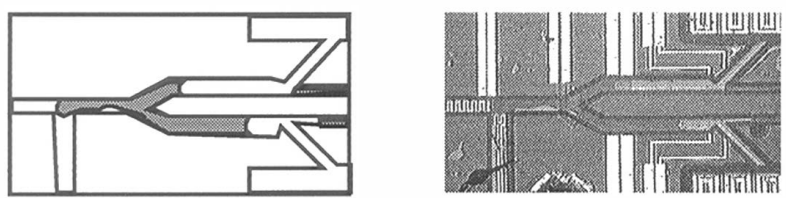

(c)
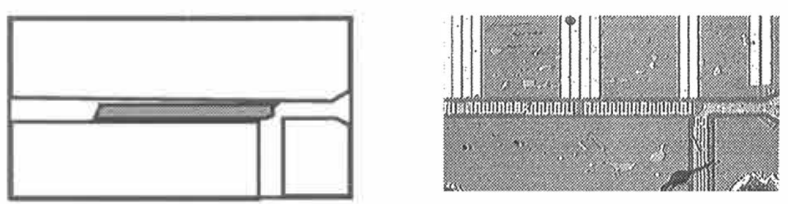

(d)

Figure 8. Schematic and photograph of the device in operation. (a): Water placed at the inlet holes are stopped at the hydrophobic patches; (b) On actuating the heaters beneath the air chambers, two discrete liquid drops ( $\sim 35 \mathrm{nl}$ each) split off; (c). 4 seconds after the heaters are turned on; $(d)$. The mixed drop is positioned beyond the hydrophobic vent.

\section{SUMMARY}

We have demonstrated the use of selective hydrophobic patterns inside microfabrifcated channel networks to control the movement of liquid samples. The air pressure required for drop splitting and motion has been generated by heating air trapped in chambers. A device has been fabricated which is used to split precisely-controlled nanoliter-volumes of different liquid samples, mix them and stop at a predetermined location. The mixed sample drop is further moved to another location by reheating the chambers after closing the vent with water.

\section{ACKNOWLEDGMENTS}

This work was supported by the National Institutes of Health under grants NIH-R01-HG01044 and NIH-R01-HG01406. We are indebted to Tim Sammarco, Brian Johnson, Sundaresh Brahmasandra and Madhavi Krishnan for their help.

\section{REFERENCES}

1. Anderson, R.C.; Bogdan, G.J.; Barniv, Z.; T.D. Dawes, J. Winkler and K. Roy, "Microfluidic Biochemical Analysis System”,Tranducers'97, pp. 477-480, 1997.

2. Burns, M.A.; Mastrangelo, C.H.; T. S. Sammarco, F.P. Man, J.R. Webster, B.N. Johnson, B. Poerster, D. Jones, Y. Fields, A.R. Kaiser and D.T. Burke, "Microfabricated Structures for Integrated DNA Analysis”, Proc. Natl. Acad. Sci., USA, vol.93, pp 5556-5561, 1996.

3. M. Koch, N. Harris, A.G.R. Evans, N.M. White and A Brunnschweiler, "A Novel Micromachined Pump Based on Thick-Film Piezoelectric Actuation”, Transducers'97, pp 353 356, 1997.

4. M. Stehr, S. Messner, H. Sandmaier, R. Zengerle; “ A New Micropump with Bidirectional Fluid Transport and Selfblocking Effect”, Proc. MEMS ‘96; pp 485-490, 1996.

5. K. Handique; B.P. Gogoi, D.T. Burke, C.H. Mastrangelo, and M.A. Burns, Microfluidic Flow Control using Selective Hydrophobic Patterning, Proceedings of SPIE Conference on Micromachined Devices and Components, Vol. 3224, pp185195, 1997.

6. K.Handique amd Mark A. Burns, unpublished work ,1998.

7. U.Srinivasan, M.R. Houston, R.T. Howe, and R. Maboudian,"Self-Assembled Fluorocarbon Films For Enhanced Stiction Reduction”, Transducers'97,pp. 1399-1402, 1997. 